\title{
OPTIMIZING PHYSICAL LAYER DATA TRANSMISSION FOR MINIMAL SIGNAL DISTORTION
}

\author{
Don H. Johnson and Hiram Rodríguez-Díaz \\ Rice University \\ Department of Electrical and Computer Engineering \\ Houston, TX 77005-1892
}

\begin{abstract}
When transmitting a sampled signal digitally, data and error correction bits must be transmitted at least as fast as the sampling rate. Typically, each bit is allocated the same transmission time interval, which means the optimal detector yields the same error probability for each bit. An alternative is to vary the bit interval duration according to the bit's contribution to the reconstructed sample. The optimal solution yields significant gains in mean-squared error (several $\mathrm{dB}$ ) over that provided by equal-duration bit intervals. These gains occurred over a wide range of signal-to-noise ratios. When block error correction is performed, we derive the optimal decoder from a Bayesian viewpoint and show that gains obtain here as well.
\end{abstract}

\section{INTRODUCTION}

In any communication system, like telephone systems, computer networks, or cellular networks, error detection and correction has been an important issue. Many different strategies have been developed for achieving the best performance of the system while using the least amount of resources. In many applications, an error in the most significant bit is far more critical than an error in the least significant bit. An error in the most significant bit greatly influences the mean-squared error of the reconstructed sample while an error in the least-significant bit has little effect. When the digital data are $K$-bit quantized signal amplitudes, the transmitted amplitude $s$ is related to the individual bits as

$$
s=\sum_{k=0}^{K-1} b_{k} 2^{k}
$$

The mean-squared distortion between the transmitted and received amplitude is, when no error correction is used, given by

$$
\text { mse }=\sum_{k=0}^{K-1} P_{e}^{(k)} 2^{2 k}
$$

where $P_{e}^{(k)}$ is the probability the $k^{\text {th }}$ bit is received in error. Thus, if the error probabilities were all equal, the most significant bit contributes $2^{2(K-1)}$ more to the mean-squared error than the least significant bit. A smaller mean-squared error could result if the error probability for the most significant bit were reduced and the error probability for the least significant bit were increased according to some kind of tradeoff.

This work was supported by the National Science Foundation under Grant CCR-0105558.

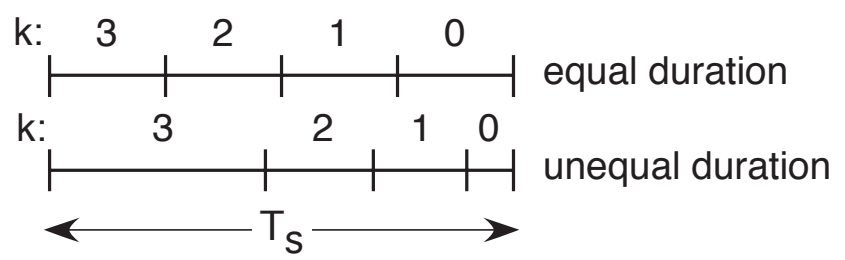

Fig. 1. Normally, bit interval durations are equal. In our proposed scheme, they differ, with much more transmission time allocated to the most significant bit (here $k=3$ ) than the least significant bit $(k=0)$. In this way, mean-squared error can be dramatically reduced. The total time to transmit each sampled signal is constrained to be no more than the sampling interval $T_{s}$.

To achieve a smaller mean-square error, one could envisage changing the error correcting code to reflect this importance, protecting more the most important bits. Our approach is along this line; but rather than a digital error correction code, we use what essentially is an analog repetition code. As shown in figure 1, we use variable-duration bit intervals to represent the various bits in the sample. Because we assume a white Gaussian noise channel and a matched filter receiver, each bit has an error probability different from that of other bits that have different transmission interval durations. Assuming a BPSK signal set, the error probability is $P_{e}^{(k)}=Q\left(\sqrt{2 A^{2} T_{k} / N_{0}}\right)$ where $A$ is the amplitude of the received sinusoidal signal and $T_{k}$ the duration of the $k^{\text {th }}$ bit interval. Longer bit intervals, which yield a smaller $P_{e}^{(k)}$, should be used for the most significant bits. The bit-interval durations are constrained since the time taken to transmit each sample must be less than or equal to the sampling interval $T_{s}$. Conseqently, as shown in figure 1 , the sum of the transmission intervals must be no more than $T_{s}$ in either the equal or unequal bit interval case. Because of the nonlinear nature of $Q(\cdot)$, how the optimal bit-interval durations should be chosen subject to this constraint is not apparent.

When digital error correction is used, it could be advantageous to allow the transmission interval durations for all bits - both data and error correction bits - to be optimized for minimum distortion. Because this would result in unequal bit error probabilities, code design and how to optimally decode need to be rethought. We take a detection theory approach here to derive an optimal decoding rule that applies whether coding is used or not. This approach yields an objective function that we seek to optimize with respect to bit interval durations constrained to sum to the sampling interval and with respect to decoding rules. 


\section{RESULTS}

We let the index $j$ denote a value for the transmitted sampled amplitude, which we take here to be an integer in the range $\left[0,2^{K-1}\right]$ (see equation 1). The decoded amplitude is indexed by $i$, and lies in the same interval. We take the distortion $D$ between the received and transmitted amplitudes to be represented by

$$
D=\sum_{i, j} \pi_{j} C_{i, j} \operatorname{Pr}[i \mid j]
$$

where $\operatorname{Pr}[i \mid j]$ is the probability that amplitude $i$ is received given that amplitude $j$ was transmitted and $\pi_{j}$ is the a priori probability that that amplitude was sent. The key quantity is the so-called Bayes' cost $C_{i, j}$, the impact of receiving amplitude $i$ when amplitude $j$ was indeed sent. Many choices for the Bayes' cost can be made. For example, if $C_{j, j}=0$ and $C_{i, j}=1$, then the nature of the amplitude error doesn't matter. On the other hand, if $C_{i, j}=(i-j)^{2}$, then mean-squared error defines the distortion. We focus on this choice here.

We assume that $N$ bits are used to transmit the $K$ data bits $(N \geq K)$. The additional $N-K$ bits provide some measure of error correction. We denote by $\mathbf{b}^{(j)}$ the transmitted $N$-bit sequence corresponding to amplitude $j$ and by $\mathbf{b}^{(i)}$ the decoded bit sequence derived from $\mathbf{b}^{(j)}$ that equals to transmitted bit-sequence corresponding to amplitude $i$. The data bits constituting the sample amplitude are derived from these. The probability that the decoder yields the bit sequence $\mathbf{b}^{(i)}$ when $\mathbf{b}^{(j)}$ was transmitted equals

$$
\operatorname{Pr}[i \mid j]=\sum_{\mathbf{b}^{(l)} \in \mathcal{R}_{i}} \prod_{n=0}^{N-1}\left[P_{e}^{(n)}\right]^{b_{n}^{(l)} \oplus b_{n}^{(j)}}\left[1-P_{e}^{(n)}\right]^{1-b_{n}^{(l)} \oplus b_{n}^{(j)}}
$$

Here, $b_{n}^{(l)}$ denotes the $n^{\text {th }}$ bit in the transmitted sequence that corresponds to the $l^{\text {th }} N$-bit sequence and $b_{n}^{(j)}$ the same bit in the transmitted amplitude $j$. The notation $b_{n}^{(l)} \oplus b_{n}^{(j)}$ means the modulo-2 sum of the bits, which equals zero when the bits agree and one when they differ. $P_{e}^{(n)}=Q\left(\sqrt{2 A^{2} T_{n} / N_{0}}\right)$ denotes the probability the $n^{\text {th }}$ bit is received in error. $T_{n}$ denotes the duration assigned to the $n^{\text {th }}$ bit. The product denotes the probability that a given bit sequence $\mathbf{b}^{(l)}$ is received. To find the amplitude, the decoder defines decoding regions $\mathcal{R}_{i}$ which includes all possible bit sequences that correspond to amplitude $i$.

To minimize the distortion, we want to chose the bit-interval durations $T_{n}$ and the decoding regions $\mathcal{R}_{i}$ that jointly minimize (3) to yield the optimal distortion $D^{*}$.

$$
\begin{aligned}
& D^{*}=\min _{\left\{\mathcal{R}_{i}\right\},\left\{T_{n}\right\}} \sum_{i, j} \pi_{j} C_{i, j} \operatorname{Pr}[i \mid j] \\
& \text { subject to } \sum T_{n}=T_{s}, \bigcup \mathcal{R}_{i}=\{\mathbf{b}\}, \bigcap_{i_{1} \neq i_{2}} \mathcal{R}_{i_{1}} \mathcal{R}_{i_{2}}=\emptyset
\end{aligned}
$$

Here, $\{\mathrm{b}\}$ denotes the set of all $N$-bit sequences and $\emptyset$ means the empty set. The latter conditions mean that the decoding regions must include every possible received bit sequence and each received sequence can belong to only one decoding region. Normally, error correcting codes and the decoders are not designed with the ultimate interpretation of the bit sequences in mind. Mathematically, the probability $\operatorname{Pr}[i \mid j]$ would be optimized separately, then distortion considered. We take the approach here of determining how the entire physical layer should be structured so that distortion is minimized.

\subsection{Uncoded case}

Expression (2) for mean-squared error can be derived when no error correcting code is used ( $N=K$ ) by explicitly using (1) that associates bits with amplitude values. Noting that $\pi_{j} \operatorname{Pr}[i \mid j]=$ $\operatorname{Pr}[i, j]$, the sum in (3) is the expected value of $C_{i, j}$. Assuming the Bayes' cost function is $(i-j)^{2}$, the mean-squared error can be written as

$$
\text { mse }=\mathcal{E}\left[\left(\sum_{k}\left(b_{k}^{(i)}-b_{k}^{(j)}\right) 2^{k}\right)^{2}\right]
$$

Upon expanding the square, the only nonzero term occurs when the $k^{\text {th }}$ bit in what is transmitted and received disagree. The crossterms between differing bits disappear because we assume a white Gaussian noise channel, which means that the optimal receiver operates on each bit interval independently of the others, and produces statistically independent bit estimates. We are left with

$$
\text { mse }=\sum_{k} \operatorname{Pr}\left[b_{k}^{(i)} \neq b_{k}^{(j)}\right] 2^{2 k}
$$

The probability in this expression is simply the probability the bit is received in error, and (2) results. In this way, a somewhat simpler expression for the distortion can be obtained from (3).

Analytically, a solution to minimizing the mean-squared error can be derived using Lagrange multipliers. The solution must satisfy the set of $K$ equations found by differentiating expression (2) plus the Lagrange penalty term $\lambda\left(\sum_{k} T_{k}-T_{s}\right)$ with respect to the bit-interval durations and setting each of the derivatives to zero.

$$
T_{k} e^{a T_{k}}=\frac{a \lambda^{2} 2^{4 k}}{8 \pi} \quad, k=0, \ldots, K-1
$$

The constant $a$ equals $2 A^{2} / N_{0}$. In our computations, we take the total time allocated to transmitting the $K$ bits, $T_{s}$, to equal 1. Consequently, $a$ is numerically equal to $E_{w} / N_{0}$, the ratio of the signal energy received during a word interval, the time taken to transmit the data, and the white noise spectral height. Because of the variable-duration bit intervals, the usual signal-to-noise ratio $E_{b} / N_{0}$ defined over a bit interval is not a constant; our SNR defined over the word interval is constant, and provides a way for comparing results. Because the left side of the equation is a monotonic function of the bit-interval durations, a unique solution exists.

Rather than solve (5) directly, we opted to solve the original optimization problem stated in (4). Optimization over the decision regions is not necessary as only data bits are sent. We numerically solved the optimization problem using Matlab's optimization program fmincon, which uses the Nelder-Mead direct search algorithm. Figure 2 shows the results for bit-interval durations and mean-squared error gain for 8-bit data. For small SNRs, transmitting only the most-significant bit minimizes the mean-squared error. The mean-squared error decreased by a few decibels for equal-duration bit intervals in this SNR range. As SNR increases, the next most significant bits are used, and the mean-squared error continues to decrease, becoming about $13 \mathrm{~dB}$ at its maximum. Maximum gain occurs when all bits are transmitted, but not when they alloted the same duration. Further SNR increases result in equal-duration bit intervals, which means that ultimately no gain $(0 \mathrm{~dB})$ occurs.

This variation of bit-interval durations and mean-squared error gain with SNR typified the behavior when smaller number of bits 

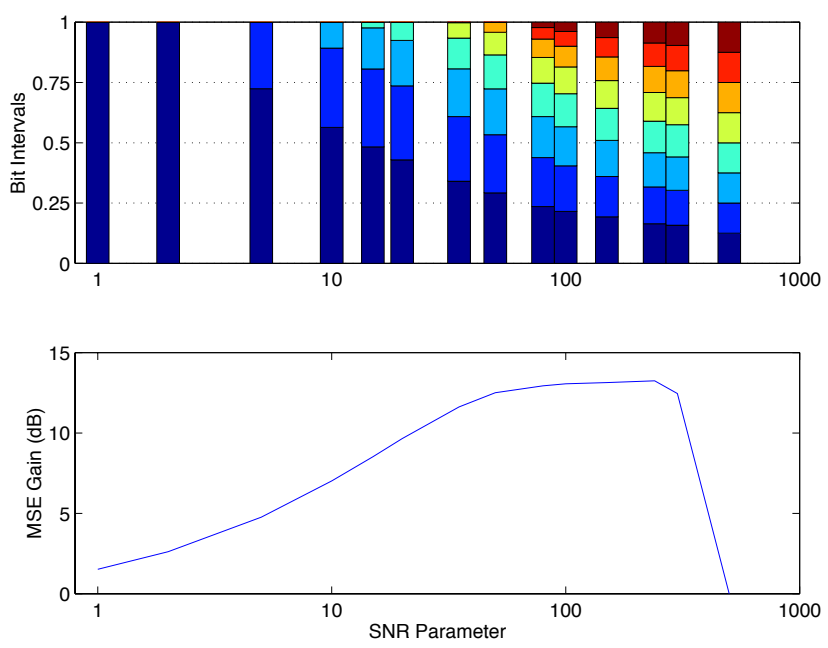

Fig. 2. The top panel shows as a stacked bar histogram the bit intervals that optimize the mean-squared error of the reconstructed data. The most-significant bit occurs at the bottom of a stack and the least-significant at the top. The horizontal axis is the signalto-noise parameter $a$ found in equation (5), which is equivalent to $E_{w} / N_{0}$. The bottom panel shows the resulting gain (decrease) in mean-squared error relative to that of equal-duration bit intervals expressed in decibels. Here, positive gains correspond to smaller mean-squared errors.

$K$ were transmitted. Figure 3 shows the mean-squared error gains that resulted. The maximal gains decrease as the number of bits decreases, and the SNR at which equal-duration bit intervals are optimal decreases. We did not consider more than eight bits.

\subsection{Coded case}

When error correction is incorporated into the communication scenario, the time taken for the coding bits can also be allowed to vary. We solved the optimization problem stated in $(4)$ for a $(7,4)$ Hamming code. The bit-interval durations of both data and errorcorrection bits were allowed to vary independently while imposing the constraint that the sum of the durations must equal the sampling interval $T_{s}$. With this constraint, the data rate equals that when no coding is used. Note that this approach means that when error correction is used, less time is available to transmit the data bits, which leads to larger bit error probabilities. This effect is countered by the ability to correct errors ( 1 error in the case of the $(7,4)$ code). However, it could well be that when the quality of the communications system is judged on the basis of signal distortion (mean-squared error), error correction may not be the best solution.

Figure 4 shows the result of our optimization. As expected, the durations of the most significant bits are longer than those of the least significant bits until large SNRs are reached. Interestingly, no error correction is optimal for SNRs smaller than a threshold (here about SNR = 60). Once this threshold is exceeded, all errorcorrection bits appear and their durations are comparable to that of the least-significant bits. Well above this threshold, equi-duration intervals are optimal. Below threshold, the gain in mean-squared error relative to the equal-duration, no-error-correction case is a few decibels larger than the gain when no error correction is used

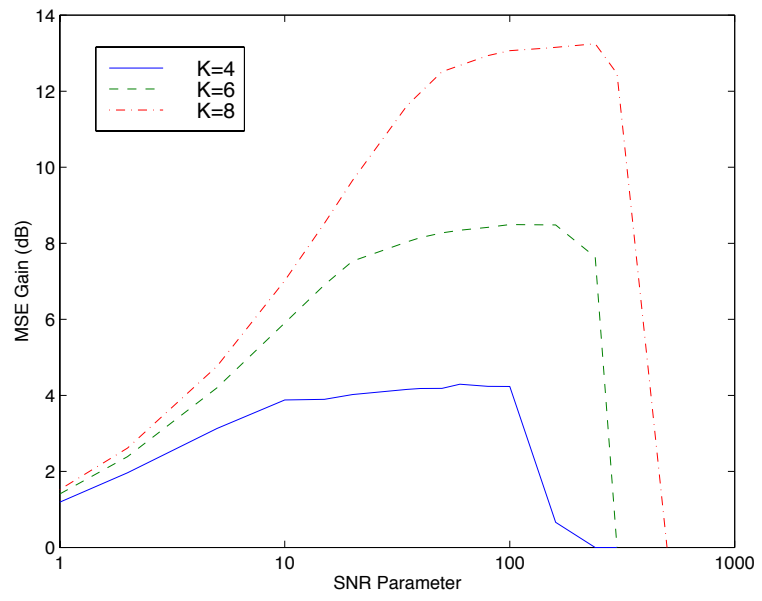

Fig. 3. The mean-squared error gain is shown for three values of $K$, the number of bits used to represent the sample's value. The mean-squared error gains decrease as fewer data bits are used. The SNR at which equal-duration bit intervals occurs decreases as the number of data bits used in the sampling decreases.
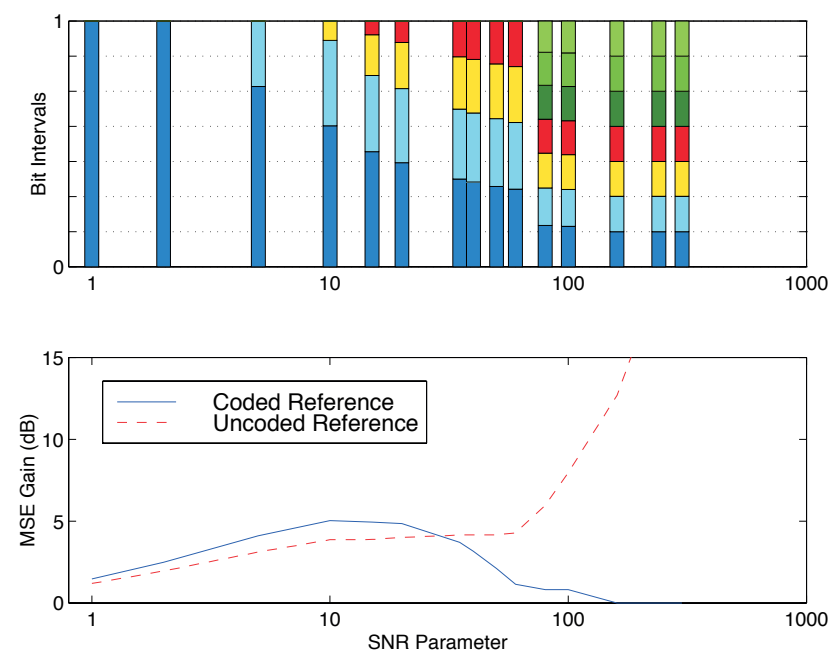

Fig. 4. The top plot shows the optimal (minimum mean-squared error) bit-interval durations as a function of $E_{w} / N_{0}$ for a $(7,4)$ Hamming code. The most significant bit lies at the botttom of each column and the least significant bit the fourth interval from the bottom. The three error-correction bit intervals are shown at the top of the columns, and only occur in the last five columns. The bottom plot shows the gains in mean-squared error that accrue in comparison to two reference values. The solid line shows the gain that results when the reference is the mean-squared error incurred by assigning equal-duration bit intervals to data and error correction bits (equal to $T_{s} / 7$ in this case). The dashed line shows the gain when the reference is equal duration bit-intervals for the no error correction case. 
(figure $3, K=4$ ). The dashed line in figure 4 shows the meansquared error achieved in comparison to the case when no errorcorrection code is used. When this gain is less than the gain over the coded case, that indicates that imposing the $(7,4)$ code actually worsens mean-squared error when bit-interval durations are equal. When the gain is higher, error-correction improves meansquared error. Error-correction improves the mean-squared error only for SNRs greater than about 40 in our example. When optimal-duration error correction is present at higher SNRs, large gains become apparent.

\section{CONCLUSIONS}

The optimality of unequal bit-interval durations for minimizing mean-squared error is not surprising. What is surprising is that the improvement in mean-squared error is so large (figure 3). Maximal gain (reduction) of mean-squared error occurs in a signal-to-noise ratio range typical of wireline communication systems (greater than $10 \mathrm{~dB}$ SNR for each bit). In this range, unequal bit-interval durations for all the data bits provides the smallest mean-squared error. For smaller SNRs more typical of wireless systems, unequal bit-interval durations and not transmitting some of the less significant bits is optimal.

The unequal bit-interval durations may be difficult to coordinate between transmitter and receiver, especially in a wireless multicast situation. That said, our results can be interpreted as how to allocate power on a bit-by-bit basis and how many bits to use to represent data. Thus, in addition to power control, additional gains can be achieved by carefully allocating transmitter power at the level of single bits.

For the single-bit block error correction considered here, folding it into the optimization was only effective at very large signalto-noise ratios. At smaller ratios, the power consumed by transmitting error correction bits is better spent on the data. The errorcorrection afforded by the code did not compensate for the increased error probability.

In summary, our results suggest that considering signal distortion at the physical as well as higher layers can be a fruitful. Our results are not tied to mean-squared error as any sample-to-sample distortion measure can be used in equation (4). 